Research Paper

\title{
The High Diversity of MRSA Clones Detected in a University Hospital in Istanbul
}

\author{
Lutfiye Oksuz ${ }^{\circledR}$, Celine Dupieux ${ }^{2}$, Anne Tristan², Michele Bes ${ }^{2}$, Jerome Etienne ${ }^{2}$, Nezahat Gurler ${ }^{1}$ \\ 1. Department of Medical Microbiology, Istanbul Medical Faculty, Istanbul University, Istanbul 34390, Turkey. \\ 2. National Reference Center for Staphylococci INSERM U851 Faculté de Médecine Lyon Est, Université de Lyon, Lyon 69008, France.
}

$\triangle$ Corresponding author: Lutfiye OKSUZ, PhD. loksuz34@yahoo.com.

(c) Ivyspring International Publisher. This is an open-access article distributed under the terms of the Creative Commons License (http://creativecommons.org/ licenses/by-nc-nd/3.0/). Reproduction is permitted for personal, noncommercial use, provided that the article is in whole, unmodified, and properly cited.

Received: 2013.04.10; Accepted: 2013.10.06; Published: 2013.10.12

\begin{abstract}
Background: To characterize the methicillin-resistant Staphylococcus aureus (MRSA) clones present in Istanbul, 102 MRSA isolates collected during a 5-year period at the Istanbul Medical Faculty Hospital were characterized using microarray analysis and phenotypic resistance profiles.

Methods: Resistance to methicillin was detected with a cefoxitin disk diffusion assay and confirmed with a MRSA-agar and MRSA detection kit. Antimicrobial susceptibility testing was performed by a disk diffusion assay and interpreted according to the 2012 guidelines of the Antibiogram Committee of the French Society for Microbiology. Decreased susceptibility to glycopeptides was confirmed using the population analysis profile-area under the curve (PAP-AUC) method. The presence of the mecA gene was detected by polymerase chain reaction. Bacterial DNA was extracted according to the manufacturer's recommended protocol using commercial extraction kits. Strains were extensively characterized using the DNA microarray.

Results: Isolates were grouped into six clonal complexes. The most frequently detected clone was the Vienna/Hungarian/Brazilian clone (ST239-MRSA-III), which accounted for $53.9 \%$ of the isolates. These isolates were resistant to multiple antibiotics, particularly penicillin, tetracycline, rifampicin, kanamycin, tobramycin, gentamicin, levofloxacin, erythromycin, lincomycin and fosfomycin. Furthermore, three isolates were detected by population analysis profile as heterogeneous vancomycin-intermediate S. aureus (hVISA). The UK-EMRSA-I5 clone (ST22-MRSA-IV PVL negative) was detected in $9.8 \%$ of the isolates and was mainly susceptible to all anti-staphylococcal antibiotics. Seven isolates $(6.9 \%)$ were positive for PVL genes and were assigned to the CC80-MRSA-IV clone (European CA-MRSA clone, three isolates), ST8-MRSA-IV clone (USA300 clone, two isolates, one ACME-positive) or ST22-MRSA-IV clone ("Regensburg EMRSA" clone, two isolates). All other clones were detected in one to six isolates and corresponded to well-known clones (e.g., Pediatric clone, Dublin EMRSA clone, WA MRSA-54/63, WA MRSA-1/57).
\end{abstract}

Conclusions: This work highlighted both the high prevalence of ST239-MRSA-III clone and the large diversity of the other MRSA clones detected in a university hospital in Istanbul.

Key words: MRSA clones, Istanbul, diversity, multiresistant ST239, PVL positive clones, ACME.

\section{BACKGROUND}

Staphylococcus aureus is both a commensal and a pathogenic bacterium that is involved in pyogenic and toxic infections. Since the early 1960s, methicillin-resistant Staphylococcus aureus (MRSA) clones have emerged and disseminated worldwide [1]. MRSA were initially associated with hospital-acquired (HA) infections and, since the 2000s, with community-acquired (CA) infections. CA-MRSA strains fre- 
quently contain the genes encoding Panton-Valentine leukocidin (PVL). MRSA clones have mainly been characterized by multilocus sequence typing (MLST) and other molecular techniques, including pulsed-field gel electrophoresis (PFGE), protein A (spa) typing and SCCmec typing. Microarray is the most recently developed tool and is well adapted to characterize MRSA clones [2,3].

In Turkey, studies of MRSA have mainly characterized the prevalence of MRSA infections and the SCCmec types of the strains, but few articles have described the molecular characteristics of MRSA. The prevalence of MRSA in Turkey varies considerably for HA-MRSA, from 12 to $75 \%$, as reported in a study performed in eight Turkish hospitals [4]. The prevalence of community-acquired MRSA appears to be low (1-3\%) in Turkey [5,6]. Both CA- and HA-MRSA strains in Turkey may contain PVL genes [7-9], and more than $80 \%$ of the strains have SCCmec type III (mainly in HA-MRSA) or, less frequently, SCCmec type IIIb, IV and V (the latter two mainly in CA-MRSA) $[4,5,7,9,10]$. Rare isolates with SCCmec type I and II have also been reported [7]. The strains with SCCmec type III are usually resistant to multiple antibiotics, in contrast to the SCCmec type IV or V strains [7]. A total of 46 MRSA strains with heteroresistance to vancomycin (hVISA) have also been reported in Turkey [11]. With respect to MRSA sequence types (STs) described in Turkey, two MRSA isolates of ST30 were characterized from nasal swabs of healthy children in a study in which 4,050 children were screened for nasal carriage of S. aureus [5], as well as 53 MRSA isolates of ST239 from human infections [4]. In a study by Turkyilmaz et al. [12], 14 and two isolates of MRSA from bovine mastitis were reported to be ST239 or ST8, respectively.

In the present study, 102 MRSA isolates collected during a 5-year period at the Istanbul Medical Faculty Hospital were characterized by microarray analysis $[2,3]$ and tested for antimicrobial susceptibility. We observed a high diversity of MRSA clones, with two prevalent clones: $53.9 \%$ of MRSA isolates were ST239-MRSA-III and 9.8\% were ST22-MRSA-IV. PVL-positive MRSA represented $6.9 \%$ of the isolates and were identified as the ST80-MRSA-IV European CA-MRSA clone (three isolates), ST8-MRSA-IV USA300 (two isolates, one ACME-positive) and ST22-MRSA-IV “Regensburg EMRSA" (two isolates).

\section{METHODS}

\section{Bacterial strains and isolates}

From September 2007 to March 2012, all MRSA isolates cultured from patient samples at the Istanbul Medical Faculty Hospital were collected. Resistance to methicillin was detected with a cefoxitin disk diffusion assay and confirmed with a MRSA-agar and MRSA detection kit (bioMérieux, Marcy l'Etoile, France). The presence of the mecA gene was detected by polymerase chain reaction (PCR) [13].

During this 5-year period, a total of 102 MRSA isolates were collected from 88 clinical samples from 85 patients. Most of the patients were male (53 of 85 , $62 \%$ ). The median age was 49 years (range, $<1$ to 90 years). Of the patients, $40(47 \%)$ were admitted at the hospital, and 45 (53\%) were from out-patient clinics. MRSA strains were isolated from skin and soft tissue infections $(54.9 \%)$, respiratory tract infections $(17.6 \%)$, bloodstream infections $(16.7 \%)$ and from other sites of infection (i.e., urinary tract, intra-abdominal, osteoarticular and ocular infections, $10.8 \%$ ).

\section{Characterization of strains by microarray}

Bacterial DNA was extracted according to the manufacturer's recommended protocol using commercial extraction kits (Qiagen, Hilden, Germany). Strains were extensively characterized using the DNA microarray Identibac S. aureus Genotyping ${ }^{\circledR}$ (Alere Technologies, Jena, Germany). This diagnostic DNA microarray, as well as related procedures and protocols, were described in detail in a previous study [3]. The microarray covers 332 different target sequences corresponding to approximately 185 distinct genes and their allelic variants and includes species markers, resistance genes, exotoxins such as PVL, MSCRAMM genes and SCCmec, capsule and agr group typing markers. The assignation of isolates to clonal complexes (CCs) was determined by comparing the hybridization profiles to previously typed MLST reference strains [2,3].

\section{Determination of antimicrobial resistance patterns}

Antimicrobial susceptibility testing was performed by a disk diffusion assay and interpreted according to the 2012 guidelines of the Antibiogram Committee of the French Society for Microbiology (CA-SFM). The following panel of antibiotics was tested: penicillin, cefoxitin, kanamycin, tobramycin, gentamicin, chloramphenicol, erythromycin, lincomycin, pristinamycin, tetracycline, rifampicin, cotrimoxazole, levofloxacin, fosfomycin, fusidic acid and linezolid.

Glycopeptide susceptibility was assessed for the most frequently detected MRSA clone (ST239-MRSA-III). Standard vancomycin and teicoplanin minimal inhibitory concentrations (MICs) were determined by a standard Etest ${ }^{\circledR}$ (bioMérieux) using a 0.5 McFarland (McF) inoculum on Mueller-Hinton agar incubated for 24 hours. Accord- 
ing to the 2012 guidelines of the CA-SFM, the breakpoint for susceptibility to glycopeptides is $2 \mathrm{mg} / \mathrm{L}$. Screening for hVISA was determined by Etest ${ }^{\circledR}$ (bioMérieux) with a $2 \mathrm{McF}$ inoculum on brain heart infusion (BHI) agar incubated for 48 hours. Visible growth at $\geq 8 \mathrm{mg} / \mathrm{L}$ for vancomycin and teicoplanin or $\geq 12 \mathrm{mg} / \mathrm{L}$ for teicoplanin only in the Etest ${ }^{\circledR}$ with a $2 \mathrm{McF}$ inoculum was considered a positive test result (14). Decreased susceptibility to glycopeptides was confirmed using the population analysis profile-area under the curve (PAP-AUC) method. The PAP-AUC was determined for selected isolates by spreading 50 $\mu \mathrm{L}$ of a $2 \mathrm{McF}$ bacterial suspension in $\mathrm{BHI}$ onto $\mathrm{BHI}$ agar plates containing increasing concentrations of vancomycin (ranging from 1 to $8 \mathrm{mg} / \mathrm{L}$ in $1 \mathrm{mg} / \mathrm{L}$ increments). After incubation at $37^{\circ} \mathrm{C}$ for 48 hours, the number of colonies was counted and graphed as $\log 10$ $\mathrm{CFU} / \mathrm{mL}$ versus the vancomycin concentration using GraphPad Prism ${ }^{\circledR}$ (GraphPad, San Diego, CA, USA) to calculate the area under the curve (AUC) [14]. ATCC29213 (MSSA strain) and Mu3 were included as control strains in each run. VISA was defined as a PAP-AUC ratio>1.3, hVISA was defined as a PAP-AUC ratio of 0.9-1.3, and susceptible MRSA was defined as a PAP-AUC ratio $<0.9$ [15].

\section{RESULTS}

A high diversity of clones was characterized by microarray, and the 102 isolates were grouped in six clonal complexes (CCs) with different prevalences (Table 1): CC8 (58.9\%), CC22 (15.7\%), CC97 (8.8\%), CC5 (7.8\%), CC1 (5.9\%) and CC80 (2.9\%). Most of the MRSA strains were of SCCmec type III (55/102, 53.9\%) or type IV $(35 / 102,34.3 \%)$. Other SCCmec types were rarely detected: type $\mathrm{V}(5 / 102,4.9 \%)$ and type Ivar $(3 / 102,2.9 \%)$. Four isolates could not be assigned a SCCmec type based on the microarray analysis; these were composite types, which correspond to unusual combinations of SCCmec elements.

The most frequently detected clone was the $\mathrm{Vi}$ enna/Hungarian/Brazilian clone (ST239-MRSA-III), which accounted for $53.9 \%$ of the isolates. A decrease of number of ST239-MRSA-III isolates was observed between the period 2007-2009 (15 to 18 per year) and the period 2010-2012 (7 to 8 per year), probably in relation with the efforts made to control MRSA spreading in hospitals. The ST239-MRSA-III clone was mostly detected in internal medicine-infectious diseases and orthopedics departments (respectively, 13 and 18 out of the 55 isolates). Isolates of this clone were of SCCmec type III and were resistant to multiple antibiotics, particularly penicillin $(100 \%)$, tetracycline $(100 \%)$, rifampicin $(100 \%)$, kanamycin, tobramycin, gentamicin (93\%), levofloxacin (93\%), erythromycin $(75 \%)$, lincomycin $(49 \%)$, fosfomycin $(58 \%)$ and fusidic acid (4\%). Various resistance genes were detected in these isolates: the bla operon, $\operatorname{erm} \mathrm{A}$, tet $\mathrm{M}$, tet efflux and fosB in all isolates (55/55, 100\%); aacA-aphD in 52/55 isolates (95\%); erm C in $27 / 55$ isolates (49\%); qac (non specific efflux pump) in 18/55 isolates (33\%); and mupR and cat in a single isolate ( $2 \%)$. Two enterotoxin genes were frequently detected in the ST239 isolates: sea (96\%) and sek-seq (86\%).

E-test screening for hVISA was positive for 19 of the 55 ST239-MRSA-III isolates (34.5\%) with teicoplanin strips (visible growth $\geq 12 \mathrm{mg} / \mathrm{L}$ ). For these 19 isolates, visible growth when using vancomycin strips ranged from 3 to $6 \mathrm{mg} / \mathrm{L}$. When using a $0.5 \mathrm{McF}$ inoculum, the median MICs to vancomycin and teicoplanin did not vary among the 19 suspected hVISA isolates and the glycopeptide-susceptible isolates. A population analysis profile was generated for 16 randomly selected ST239-MRSA-III isolates, which included eight suspected hVISA isolates selected by E-test screening and eight other isolates that were not hVISA by E-test screening, without induction by vancomycin, resulting in the detection of a single hVISA isolate (isolate ST20112190, PAP-AUC ratio 0.99 ). The same experiment was performed for additional eight isolates, including four suspected hVISA isolates and four non-suspected hVISA isolates, excluding the isolate ST20112190, after induction by 2 $\mathrm{mg} / \mathrm{L}$ vancomycin in BHI for 72 hours; heterogeneous resistance to vancomycin occurred in two of the eight isolates (isolates ST20112172 and 20112135, PAP-AUC ratios 1.00 and 1.03 , respectively).

All other clones were detected in less than $10 \%$ of the isolates but had high diversity (Table 1). The UK-EMRSA-15 clone (ST22-MRSA-IV PVL-negative) was detected in $9.8 \%$ of the isolates. The temporal distribution of ST22-MRSA-IV isolates was quite stable during the study time (2 to 5 isolates per year). These isolates were mostly isolated in pediatric wards (5 out of the 10 isolates). UK-EMRSA-15 isolates were of SCCmec type IV and were mainly susceptible to all anti-staphylococcal antibiotics with the exception of one isolate that was resistant to tetracycline (encoded by the tetK gene). All ST22 isolates were positive for the bla operon and the enterotoxin gene cluster $(\mathrm{egc})$, and two were positive for the tst- 1 gene.

Of the 102 isolates, seven $(6.9 \%)$ were positive for PVL genes and were assigned to three clones: the CC80-MRSA-IV clone (European CA-MRSA clone) for three isolates, the ST8-MRSA-IV clone (USA300 clone) for two isolates and the ST22-MRSA-IV clone ("Regensburg EMRSA" clone) for two isolates. All PVL-positive MRSA strains were isolated from skin and soft tissue infections, with the exception of an isolate cultured from a transtracheal aspiration. 
Table I. Distribution and characteristics of the I02MRSA isolates collected at the Istanbul Medical Faculty Hospital between September 2007 and March 2012.

\begin{tabular}{|c|c|c|c|c|c|c|c|c|}
\hline $\begin{array}{l}\text { CC (number } \\
\text { of isolates) }\end{array}$ & $\begin{array}{l}\text { MLST assignment } \\
\text { (Alere) }^{*}\end{array}$ & Clone assignment (Alere)* & $\begin{array}{l}\text { Number of } \\
\text { isolates }\end{array}$ & $\begin{array}{l}\% \text { of } \\
\text { isolates }\end{array}$ & $\begin{array}{l}\text { agr } \\
\text { type }\end{array}$ & SCCmec type & PVL & $\begin{array}{l}\text { Antibiotic } \\
\text { resistance } \geq 90 \% * *\end{array}$ \\
\hline \multirow[t]{3}{*}{ CC8 (60) } & $\mathrm{CC} 8$ & ST8-MRSA-IV, USA300 & 2 & 2.0 & 1 & IV & + & Kan, Ery, Lvx \\
\hline & CC8/ST239 & $\begin{array}{l}\text { ST239-MRSA-III, Vien- } \\
\text { na/Hungarian/Brazilian Clone }\end{array}$ & 55 & 53.9 & 1 & III & - & $\begin{array}{l}\text { Kan, Tob, Gen, Tet, } \\
\text { Rif, Lvx }\end{array}$ \\
\hline & CC8/ST72 & ST72-MRSA-IV, USA700 & 3 & 2.9 & 1 & IV & - & Tet, Fus \\
\hline \multirow[t]{5}{*}{ CC22 (16) } & $\mathrm{CC} 22$ & $\begin{array}{l}\text { ST22-MRSA-IV [PVL+], "Regens- } \\
\text { burg EMRSA" }\end{array}$ & 2 & 2.0 & 1 & IV & + & Kan, Tob, Ery, Lvx \\
\hline & $\mathrm{CC} 22$ & ST22-MRSA-IV, "Dublin EMRSA" & 1 & 1.0 & 1 & IV & - & \\
\hline & $\mathrm{CC} 22$ & $\begin{array}{l}\text { ST22-MRSA-IV, } \\
\text { Barnim/UK-EMRSA-15 }\end{array}$ & 10 & 9.8 & 1 & IV & - & \\
\hline & $\mathrm{CC} 22$ & ST22-MRSA-V & 2 & 2.0 & 1 & $\mathrm{~V}$ & - & \\
\hline & $\mathrm{CC} 22$ & None & 1 & 1.0 & 1 & Composite ${ }^{* * *}$ & - & Ery, Fus \\
\hline \multirow[t]{2}{*}{ CC97 (9) } & CC97 & CC97-MRSA-IV, WA MRSA-54/63 & 6 & 5.9 & 1 & IV & - & \\
\hline & CC97 & None & 3 & 2.9 & 1 & Composite ${ }^{* * *}$ & - & \\
\hline \multirow[t]{3}{*}{ CC5 (8) } & CC5 & $\begin{array}{l}\text { CC5-MRSA-IV, Pediatric clone } \\
\text { [sed/j/r-negat. subclone] }\end{array}$ & 3 & 2.9 & 2 & IV & - & Ery, Lin, Lvx \\
\hline & CC5 & $\begin{array}{l}\text { CC5-MRSA-Ivar., WA } \\
\text { MRSA-18/21/48 }\end{array}$ & 3 & 2.9 & 2 & Ivar & - & Kan, Tob \\
\hline & $\mathrm{CC} 5$ & $\begin{array}{l}\text { CC5-MRSA-V [sed/j/r-posit. sub- } \\
\text { clone], WA MRSA-11/34/35/87 }\end{array}$ & 2 & 2.0 & 2 & $\mathrm{~V}$ & - & \\
\hline \multirow[t]{2}{*}{ CC1 (6) } & $\mathrm{CC} 1$ & CC1-MRSA-IV, WA MRSA-1/57 & 5 & 4.9 & 3 & IV & - & \\
\hline & $\mathrm{CC} 1$ & CC1-MRSA-V & 1 & 1.0 & 3 & $\mathrm{~V}$ & - & Ery, Lvx \\
\hline CC80 (3) & CC80 & $\begin{array}{l}\text { CC80-MRSA-IV [PVL+], European } \\
\text { CA-MRSA Clone }\end{array}$ & 3 & 2.9 & 3 & IV & + & Kan, Fus \\
\hline Total & & & 102 & 100.0 & & & & \\
\hline
\end{tabular}

The three isolates of the European CA-MRSA clone were resistant to penicillin, kanamycin and fusidic acid. Two were also resistant to tetracycline, while the third was susceptible to tetracycline and resistant to rifampicin. All were positive for the bla operon and the resistance genes for aminoglycosides and tetracycline (aphA3, sat, tet $\mathrm{K}$ and tet Efflux) as well as for the etd gene. The two isolates of the USA300 clone were resistant to penicillin, kanamycin, erythromycin and levofloxacin and were positive for the bla operon and genes encoding resistance to macrolides ( $m s r \mathrm{~A}$ and $m p b \mathrm{BM})$, aminoglycosides (aph $\mathrm{A} 3$ and sat), and other antibiotics (fosB and tet Efflux genes), as well as the sek-seq genes. One of the USA300 clone isolates harbored the arginine catabolic mobile element (ACME) gene. The two isolates of Regensburg EMRSA (ST22-MRSA-IV PVL-positive) were resistant to penicillin, kanamycin, tobramycin, erythromycin and levofloxacin, and one of the two isolates was also resistant to gentamicin. Resistance was encoded by the erm $\mathrm{C}$ gene for erythromycin, by the aac $\mathrm{A}-a p h \mathrm{D}$ and aad $\mathrm{D}$ genes for aminoglycosides and by the bla operon for penicillin.

All other clones were detected in one to six iso- lates and corresponded to uncharacterized clones (one CC22 isolate and three CC97 isolates), to well-known clones such as the Pediatric clone, or to other described clones such as the Dublin EMRSA clone, WA MRSA-54/63 or WA MRSA-1/57.

\section{DISCUSSION}

In this study, microarray analysis of 102 MRSA isolates collected in an Istanbul hospital revealed a high diversity of MRSA clones, with a predominance of two clones: the Vienna/Hungarian/Brazilian clone ST239 (accounting for $53.9 \%$ of the isolates), which is a well-known multi-drug resistant HA-MRSA clone, and the HA-MRSA clone ST22-MRSA-IV Barnim/UK-EMRSA-15 (accounting for $9.8 \%$ of the isolates). Prior to this study, ST22 had not been described in Turkey. This study also revealed the presence of the PVL genes in $6.9 \%$ of the isolates.

The major limitation of this epidemiological study of MRSA clones is its restriction to a single hospital of Istanbul. This study does not reflect the overall MRSA epidemiology in Turkey but clearly indicates the high diversity of MRSA clones of different geographic origins in a single hospital. This 
diversity can be attributed to the demographic and geographic characteristics of Istanbul, a city of approximately 15 million of inhabitants at a crossroad between Europe and Asia and a center of international touristic and economic exchange. The diversity of MRSA clones detected in Istanbul is similar to that reported for other cities that are considered hubs of exchange for MRSA clones, such as Singapore [16].

In Turkey, few articles have described the molecular characteristics of MRSA. In the one of these studies, van Belkum et al [29] showed that a major MRSA clone circulated in hospitals in Bursa and Ankara cities of Turkey. The authors also reported that the strains from Istanbul had genetic resemblance to those from Ankara according to preliminary data.

The ST239-MRSA-III lineage was detected in $53.9 \%$ of the Istanbul isolates and is a major pandemic MRSA clone that is frequently isolated in Turkey, Iran, Saudi Arabia, Hong Kong and China [2]. It belongs to clonal complex 8 (CC8) and resulted from the transfer of an approximately $600-\mathrm{kb}$ fragment of the ST30 genome into the CC8 parent strain $[1,2]$. Complete genome sequences of four ST239 strains have already been determined (TW20 in London, JKD6008 in New Zealand, T0131 in China and 16K in Russia), revealing differences in both genomic and mobile genetic elements. Harris et al. studied the geographic structure and evolution of ST239 isolates by analyzing genome-wide single-nucleotide polymorphisms [17]. The three Turkish isolates included in the Harris et al. study were shown to belong to the European clade, which is considered the ancestral clade and has a common ancestor dating to the mid-1950s [18] or the 1960s [17], and not to the South-American or Asian clades. The worldwide distribution of ST239-MRSA-III explains the different names of this clone (e.g., Brazilian, EMRSA-1, -4, -7, -9, -11, CMRSA-3, Czech, Hungarian or Vienna clones) [17, 18]. It is a HA-MRSA clone that is resistant to multiple antibiotics, allowing it to disseminate in hospital environments. Various resistance genes have been described in ST239 isolates for macrolides (ermA, erm C), aminoglycosides (aacA-aphD, aadD, aphA3, sat), trimethoprim (dfrA), mupirocin (mupA) and tetracyclines (tetK) as well as to antiseptics [2]. In our study, we identified similar genes: erm $\mathrm{A}$, tet $\mathrm{M}$, tet efflux and fosB in all isolates $(55 / 55,100 \%)$; aacA-aph $\mathrm{D}$ in $52 / 55$ isolates (95\%); erm C in 27/55 isolates (49\%); qac (nonspecific efflux pomp) in 18/55 isolates (33\%); and mup R and cat in a single isolate ( $2 \%)$. Note that some genes, such as erm $\mathrm{A}$ and fos $\mathrm{B}$, were frequently detected but were not systematically expressed when evaluated for an antibiotic resistance phenotype. In a study by van Hal et al. [19], hVISA isolates were detected by PAP-AUC in 46/311 (14.8\%) of the ST239 isolates and not in the other clones. In our study, the investigation of hVISA isolates of ST239 was conducted with a limited number of isolates with and without induction by vancomycin. Three isolates were considered hVISA by PAP-AUC, but the incidence of hVISA was not determined because we did not perform PAP-AUC for all ST239 isolates. In Turkey, Sancak et al. reported that $18 \%$ of the 256 MRSA strains in their study were hVISA [11]. hVISA strains of the ST239-MRSA-III clone have been shown to harbor a mutation in the walKR locus, a two-component regulatory locus involved in drug resistance and metabolic control (20). PVL was not observed in ST239 isolates in the current study as in previous studies [2].

The ST22-MRSA-IV was detected in $9.8 \%$ of the Turkish isolates and is also a pandemic HA-MRSA clone. It has been described in many countries and is known as UK-EMRSA-15, Irish AR06, Barnim epidemic strain, Spanish PFGE type E13 or Canadian MRSA-8 [2]. This clone has been associated with both hospital- and community-acquired infections as well as with domestic animals such as dogs, cats and horses. Its higher frequency observed in pediatric wards in this study had not been previously reported. ST22-MRSA-IV often harbors the $\beta$-lactamase and erm $\mathrm{C}$ genes [2]. In this study, we did not detect erythromycin resistance in the Turkish ST22-MRSA-IV PVL-negative isolates, but erythromycin resistance was detected in the two ST22-MRSA-IV PVL-positive isolates. Variable virulence genes were also detected; the egc locus and sec-sel genes were frequently detected, while the sea and tst-1 genes were detected less frequently [2]. The Turkish ST22 isolates were all positive for the egc locus, and two of ten were positive for the tst-1 gene.

The presence of PVL genes in Turkey ranges from 0 to $12.7 \%$ [7-10, 27, 28]. In this study, PVL was detected in $6.9 \%$ of the isolates, and these isolates belonged to three CA-MRSA clones. This proportion is low, but the diversity of the PVL-positive isolates must be highlighted. The ST8-MRSA-IV USA300 clone is more frequently detected in Spain [21] and North [22] and South America [23]. The ST80 clone is considered the major European CA-MRSA clone $[13,24]$ and is also frequently detected in Algeria [25]. The isolation of ST80-MRSA-IV from human infections in Turkey had not been previously described. The ST22-MRSA-IV Regensburg clone has been reported in two nosocomial outbreaks and in community-acquired infections in Southern Germany. PVL-positive ST22 strains have been detected in patients living in Germany and originating from Turkey, or having travelled to England [26]. 


\section{CONCLUSIONS}

In summary, this microarray-based study highlights the great diversity of MRSA clones present in the Istanbul Medical Faculty Hospital.

\section{Acknowledgements}

We would like to thank to American Journal Experts who analysed English grammar and made significant revision of the manuscript. This study was supported by "The Scientific Research Projects Coordination Unit of Istanbul University" (Project number: 2836).

\section{Author's contributions}

$\mathrm{LO}, \mathrm{MB}$ and AT carried out the initial conception of the study, analysis and interpretation of data, revision of the different drafts. CD involved in data collection, analysis and interpretation of data, writing and revision. JE and NG critically revised the manuscript and carried out the final corrections. All authors read and approved the final manuscript.

A part of this study was presented at $22^{\text {nd }}$ European Congress of Clinical Microbiology and Infectious Diseases (ECCMID) in London (Poster No: 1401).

\section{Ethical standards}

The approval of an ethics committee is not necessary, because the clinical samples of our study were taken as part of standard patient care.

\section{Competing interests}

The authors have declared that no competing interest exists.

\section{References}

1. Deurenberg RH, Stobberingh EE. The evolution of Staphylococcus aureus. Infect Genet Evol. 2008; 8: 747-63.

2. Monecke S, Coombs G, Shore AC, Coleman DC, Akpaka P, Borg M, Chow H, Ip M, Jatzwauk L, Jonas D, Kadlec K, Kearns A, Laurent F, O'Brien FG, Pearson J, Ruppelt A, Schwarz S, Scicluna E, Slickers P, Tan HL, Weber S, Ehricht R. A field guide to pandemic, epidemic and sporadic clones of methicillin-resistant Staphylococcus aureus. PLoS One. 2011; 6: e17936.

3. Monecke S, Luedicke C, Slickers P, Ehricht R. Molecular epidemiology of Staphylococcus aureus in asymptomatic carriers. Eur J Clin Microbiol Infect Dis. 2009; 28: 1159-65.

4. Alp E, Klaassen CH, Doganay M, Altoparlak U, Aydin K, Engin A, Kuzucu C, Ozakin C, Ozinal MA, Turhan O, Voss A. MRSA genotypes in Turkey: persistence over 10 years of a single clone of ST239. J Infect. 2009; 58: 433-8.

5. Kilic A, Mert G, Senses Z, Bedir O, Aydogan H, Basustaoglu AC, Appelbaum PC. Molecular characterization of methicillin-resistant Staphylococcus aureus nasal isolates from Turkey. Antonie Van Leeuwenhoek. 2008; 94: 615-9.

6. Cesur S, Cokca F. Nasal carriage of methicillin-resistant Staphylococcus aureus among hospital staff and outpatients. Infect Control Hosp Epidemiol. 2004; 25: 169-71.

7. Kilic A, Guclu AU, Senses Z, Bedir O, Aydogan H, Basustaoglu AC. Staphylococcal cassette chromosome mec (SCCmec) characterization and panton-valentine leukocidin gene occurrence for methicillin-resistant Staphylococcus aureus in Turkey, from 2003 to 2006. Antonie Van Leeuwenhoek. 2008; 94: 607-14.

8. Akoglu H, Zarakolu P, Altun B, Unal S. Epidemiological and molecular characteristics of hospital-acquired methicillin-resistant Staphylococcus aureus strains isolated in Hacettepe university adult hospital in 2004-2005. Mikrobiyol Bul. 2010; 44: 343-55.
9. Baran CB, Mutlu D, Baysan BO, Gunseren F, Ergani A, Ogunc D, Colak D. Investigation of Panton-Valentine leukocidin gene, SCCmec gene cassette types and genotypes of methicillin-resistant Staphylococcus aureus strains isolated from outpatients. Mikrobiyol Bul. 2010; 44: 533-45.

10. Karahan ZC, Tekeli A, Adaleti R, Koyuncu E, Dolapci I, Akan OA. Investigation of Panton-Valentine leukocidin genes and SCCmec types in clinical Staphylococcus aureus isolates from Turkey. Microb Drug Resist. 2008; 14: 203-10.

11. Sancak B, Ercis S, Menemenlioglu D, Colakoglu S, Hascelik G. Methicillin-resistant Staphylococcus aureus heterogeneously resistant to vancomycin in a Turkish university hospital. J Antimicrob Chemother. 2005; 56: 519-23.

12. Turkyilmaz S, Tekbiyik S, Oryasin E, Bozdogan B. Molecular epidemiology and antimicrobial resistance mechanisms of methicillin-resistant Staphylococcus aureus isolated from bovine milk. Zoonoses Public Health. 2008; 57: 197-203.

13. Dauwalder O, Lina G, Durand G, Bes M, Meugnier H, Jarlier V, Coignard B, Vandenesch F, Etienne J, Laurent F. Epidemiology of invasive methicillin-resistant Staphylococcus aureus clones collected in France in 2006 and 2007. J Clin Microbiol. 2008; 46: 3454-8.

14. Wootton M, Howe RA, Hillman R, Walsh TR, Bennett PM, MacGowan AP. A modified population analysis profile (PAP) method to detect hetero-resistance to vancomycin in Staphylococcus aureus in a UK hospital. J Antimicrob Chemother. 2001; 47: 399-403.

15. Khatib R, Jose J, Musta A, Sharma M, Fakih MG, Johnson LB, Riederer K, Shemes S. Relevance of vancomycin-intermediate susceptibility and heteroresistance in methicillin-resistant Staphylococcus aureus bacteraemia. J Antimicrob Chemother. 2011; 66: 1594-9.

16. Hsu LY, Tristan A, Koh TH, Bes M, Etienne J, Kurup A, Tan TT, Tan BH. Community associated methicillin-resistant Staphylococcus aureus, Singapore. Emerg Infect Dis. 2005; 11: 341-2.

17. Harris SR, Feil EJ, Holden MT, Quail MA, Nickerson EK, Chantratita N, Gardete S, Tavares A, Day N, Lindsay JA, Edgeworth JD, de Lencastre H, Parkhill J, Peacock SJ, Bentley SD. Evolution of MRSA during hospital transmission and intercontinental spread. Science. 2010; 327: 469-74.

18. Smyth DS, McDougal LK, Gran FW, Manoharan A, Enright MC, Song JH, de Lencastre $\mathrm{H}$, Robinson DA. Population structure of a hybrid clonal group of methicillin-resistant Staphylococcus aureus, ST239-MRSA-III. PLoS One. 2010; 5: e8582.

19. van Hal SJ, Jones M, Gosbell IB, Paterson DL. Vancomycin heteroresistance is associated with reduced mortality in ST239 methicillin-resistant Staphylococcus aureus blood stream infections. PLoS One. 2011; 6: e21217.

20. Howden BP, McEvoy CR, Allen DL, Chua K, Gao W, Harrison PF, Bell J, Coombs G, Bennet-Wood V, Porter JL, Robins-Browne R, Davies JK, Seemann $\mathrm{T}$, Stinear TP. Evolution of multidrug resistance during Staphylococcus aureus infection involves mutation of the essential two component regulator WalKR. PLoS Pathog. 2011; 7: e1002359.

21. Blanco R, Tristan A, Ezpeleta G, Larsen AR, Bes M, Etienne J, Cisterna R, Laurent F. Molecular epidemiology of Panton-Valentine leukocidin-positive Staphylococcus aureus in Spain: emergence of the USA300 clone in an autochthonous population. J Clin Microbiol.2011; 49: 433-6.

22. Klevens RM, Morrison MA, Nadle J, Petit S, Gershman K, Ray S, Harrison LH, Lynfield R, Dumyati G, Townes JM, Craig AS, Zell ER, Fosheim GE, McDougal LK, Carey RB, Fridkin SK; Active Bacterial Core surveillance (ABCs) MRSA Investigators. Invasive methicillin-resistant Staphylococcus aureus infections in the United States. JAMA. 2007; 298: 1763-71.

23. Reyes J, Rincon S, Diaz L, Panesso D, Contreras GA, Zurita J, Carrillo C, Rizzi A, Guzman M, Adachi J, Chowdhury S, Murray BE, Arias CA. Dissemination of methicillin-resistant Staphylococcus aureus USA300 sequence type 8 lineage in Latin America. Clin Infect Dis. 2009; 49: 1861-7.

24. Otter JA, French GL. Molecular epidemiology of community-associated methicillin-resistant Staphylococcus aureus in Europe. Lancet Infect Dis. 2010; 10: 227-39.

25. Antri K, Rouzic N, Dauwalder O, Boubekri I, Bes M, Lina G, Vandenesch $\mathrm{F}$, Tazir M, Ramdani-Bouguessa N, Etienne J. High prevalence of methicillin-resistant Staphylococcus aureus clone ST80-IV in hospital and community settings in Algiers. Clin Microbiol Infect. 2010; 17: 526-32.

26. Maier J, Melzl H, Reischl U, Drubel I, Witte W, Lehn N, Linde H. Panton-Valentine leukocidin-positive methicillin-resistant Staphylococcus aureus in Germany associated with travel or foreign family origin. Eur J Clin Microbiol Infect Dis. 2005; 24: 637-9.

27. Tekeli A, Koyuncu E, Dolapçi I, Akan OA, Karahan ZC. Molecular characteristics of methicillin-resistant Staphylococcus aureus strains isolated from blood cultures between 2002-2005 in Ankara University Hospital. Mikrobiyol Bul. 2009; 43: 1-10.

28. Kirdar S, Arslan U, Tuncer I, Findik D, Bozdoğan B. Investigation of the clonality and Panton-Valentine leukocidin toxin among nosocomial methicillin-resistant Staphylococcus aureus strains. Mikrobiyol Bul. 2009; 43: 529-33.

29. van Belkum A, van Leeuwen W, Verkooyen R, Saçilik SC, Cokmus C, Verbrugh H. Dissemination of a single clone of methicillin-resistant Staphylococcus aureus among Turkish hospitals. J. Clin. Microbiol. 1997; 35(4):978. 